\title{
FRACTURES OF THE RADIUS AND ULNA*
}

\author{
E. Mervyn Evans, Swansea, Walfs \\ Formerly of the Birmingham Accident Hospital
}

Fractures of the radius and ulna without displacement, incomplete fractures of both bones and isolated fractures of the shaft of the ulna seldom present any difficulties in treatment. On the other hand complete fractures of the shaft of the radius and fractures of both bones with displacement are much more difficult injuries and many cause anxiety during treatment and dissatisfaction with the final result. This paper is mainly concerned with these more difficult injuries and is based on a study of fifty consecutive displaced fractures of both bones of the forearm treated by conservative methods. Certain aspects of the mechanism and treatment of greenstick fractures of the forearm are also discussed.

\section{COMPLETE FRACTURES OF THE RADIUS AND ULNA}

Complete fractures of the shaft of the radius, and especially fractures of both bones of the forearm, have hitherto been regarded as so difficult to reduce and hold reduced that many surgeons advise immediate open reduction and internal fixation, at any rate in the adult.

To discover why these fractures are so refractory it is necessary to

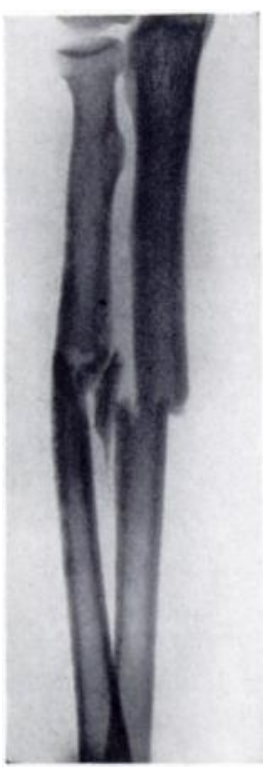

FIG. 1

The discrepancy in width between the tworadial fragments indicates that a rotation deformity. is present. consider some aspects of the anatomy of the forearm. The shape of the forearm is of itself a cause of difficulty; the radius lies deep beneath muscles and fascial compartments, and although the ulna is subcutaneous it is not easy to manipulate. The fact that there are two bones to set and hold at the same time accounts for some of the failures. But the main difficulty lies in the element of rotation, which is of peculiar importance in the forearm yet has been largely ignored.

In pronation and supination the head of the radius pirots in the annular ligament while the lower end swings around the head of the ulna. At the moment of a fracture of both bones of the forearm the continuity between the proximal and distal pivots is broken and the upper radial fragment is rotated into a position of equilibrium by the soft-tissue structures attached to it, leaving the lower fragment of the radius behind with the two ulnar fragments. Thus a deformity is produced between the upper radial fragment and the rest of the forearm-a rotational deformity often as much as 90 degrees, which demands correction no less than do shortening and angulation. It is not surprising that our results are imperfect if we ignore this, the most important element in the function of the forearm. Rotational deformity between the two fragments of the radius can occur whatever the level of the fracture. It is frequently obvious on the first radiograph from a discrepancy in the width of the two radial fragments (Fig. 1) and it can be observed in old fractures which have been allowed to unite in an incorrect rotational position Rotation does not occur between the two fragments of the ulna; it is presumably prevented by the soft tissues attached to them. It may be argued that the rotational position of the proximal radial fragment is not static, that further rotation may occur during reduction or while in plaster, and that the determination of the rotational position at the time of

* Based on a Hunterian Lecture delivered at the Royal College of Surgeons of England on February 28, 1950. 
admission is therefore of no value. This is not so: at the moment of fracture the upper radial fragment is pulled into a certain position of rotation by the soft-tissue forces acting upon it, and it retains this position throughout. Find this position at the outset and place the rest of the forearm in alignment with it, and one has solved the problem of rotational deformity in that particular fracture (Fig. 2). In only one of the series of fifty cases to be described was there a significant discrepancy in the rotational position at the time of reduction and that at the end of treatment. In the remaining forty-nine patients the original rotational deformity was corrected to within a few degrees and was held throughout treatment. Further evidence of the rotational stability of the upper fragment may be obtained by rotating it under direct vision during operations on open fractures. It can be rotated fairly easily but always returns to its original position. Determination of rotational deformity-It is an elementary principle in fracture reduction that when the position of one fragment cannot be controlled the remainder of the part must be brought into line with it. In forearm fractures the rotation of the proximal radial fragment is uncontrollable; its position in space must therefore be determined so that the remainder of the limb may be lined up with it. This is done by studying the shape of the bicipital tuberosity (as seen in anteroposterior radiographs) and comparing it with that of the normal side in varying degrees of rotation. In full supination the bicipital tuberosity is prominent on the ulnar side of the radius; in mid-rotation it has moved to the back of the bone and is not visible as a projection; and in full pronation it appears as a projection on the lateral side. Between these positions there are fine grades of difference in shape and prominence which can be used as a guide, accurate to within a few

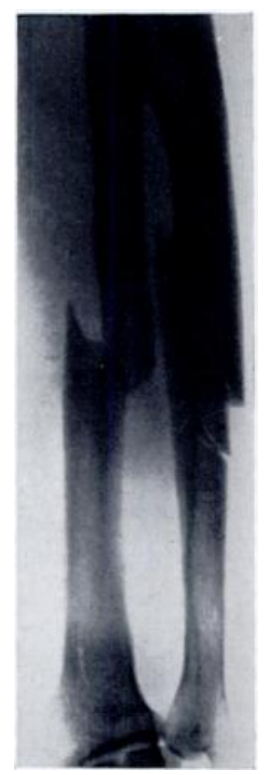

FIG. 2

A fracture of both bones of the forearm before (Fig. 2) and after (Fig. 3) correction of rotational deformity: degrees, for determining the rotational position of the radius. In practice the appearances that matter are those between mid-rotation and full supination, for after a fracture of the radius the upper fragment is seldom found on the pronation side of the neutral position. The shape and texture of the tuberosity vary in different patients and at different ages, but in any one patient the tuberosity is identical on the two sides.

Technique - To achieve standardisation a constant technique must be employed to demonstrate what may be termed the "tuberosity view"; the method of taking this view (Evans 194.5) is briefly as follows. An antero-posterior radiograph of the bicipital tuberosity on the injured side is made, and compared with antero-posterior views of the normal radius taken with the forearm in neutral rotation, 30 degrees supination, and 60 degrees supination. In high fractures of the radius and in cases in which the rotation on the injured side seems to be more than 60 degrees of supination, a further view of the normal side in full supination is also taken. Comparison of the outline of the tuberosity on the injured side with that of the normal tuberosity in known degrees of rotation gives an accurate idea of the rotation of the proximal fragment of the radius (Figs. 4 to 7 ).

In matching the normal with the injured side several characteristics in the appearance of the tuberosity may be taken into account (Fig. 8): 1) In full supination the tuberosity is usually most prominent and its curve is short and pronounced. As the mid-position is approached the tuberosity becomes progressively less prominent and its curve larger and more gradual. 2) In full supination the cancellous bone of the tuberosity may be seen at the extreme 


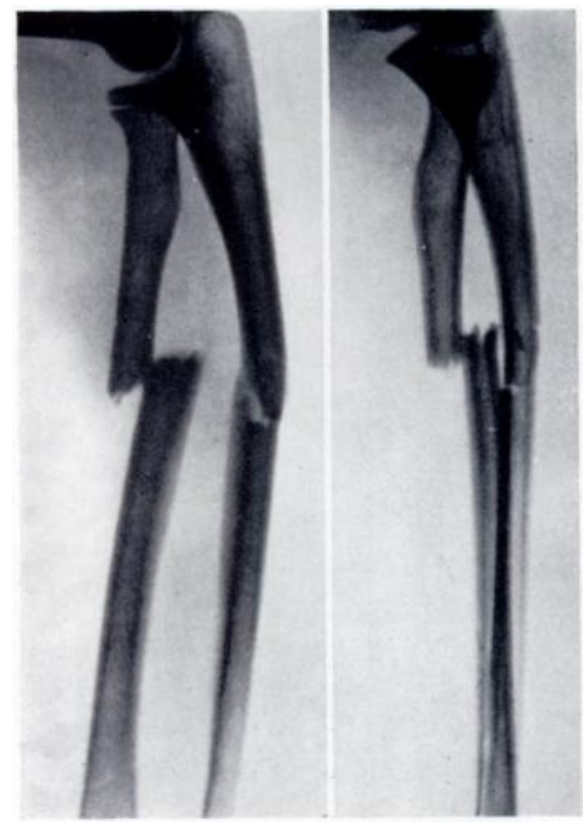

Fig. 4

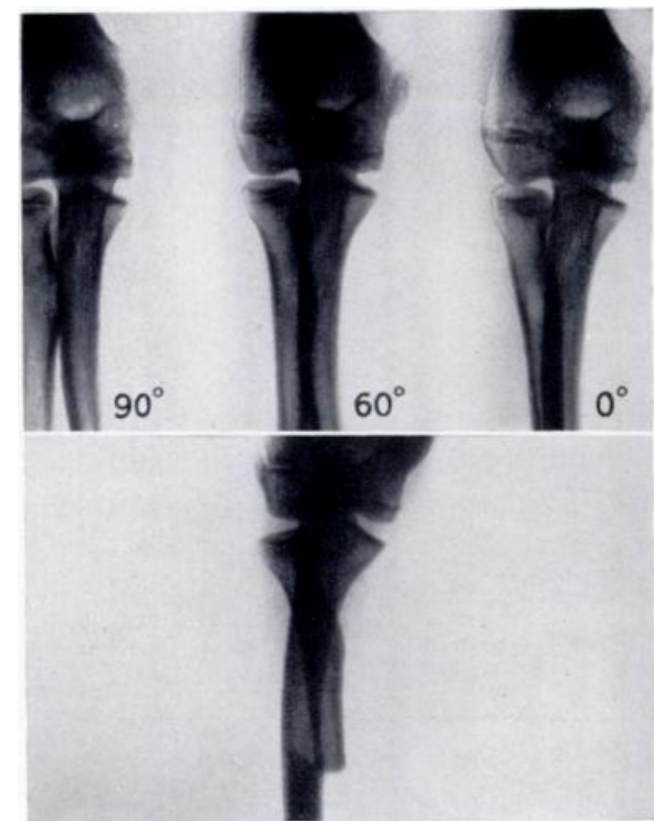

FIG. 5

Fracture of both bones in the forearm. Figure 4-Initial radiograph. Figure 5- (fbore) The normal forearm in neutral rotation, in 60 degrees supination and in 90 degrees supination. (B'lo'i') The injured forearm. The shape of the bicipital tuberosity on the injured side corresponds with that of the normal side with the forearm supinated 60 degrees.

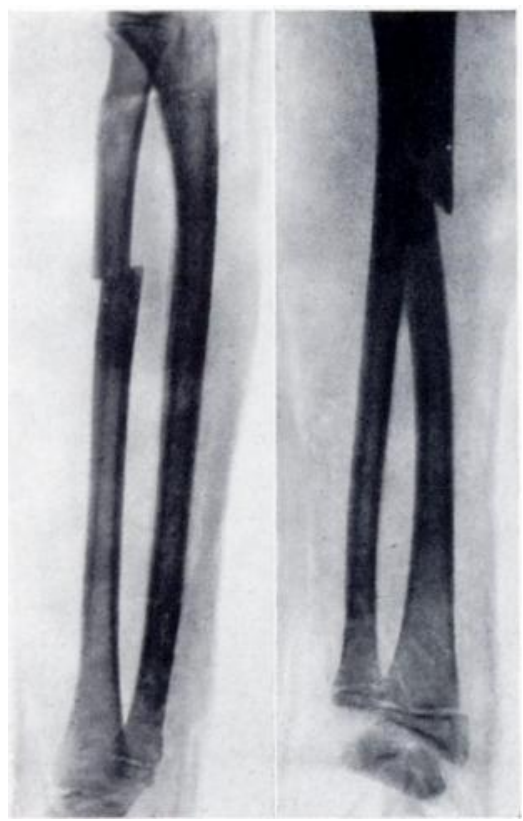

FIG. 6

The fracture is reduced after supinating the forearm 60 degrees.

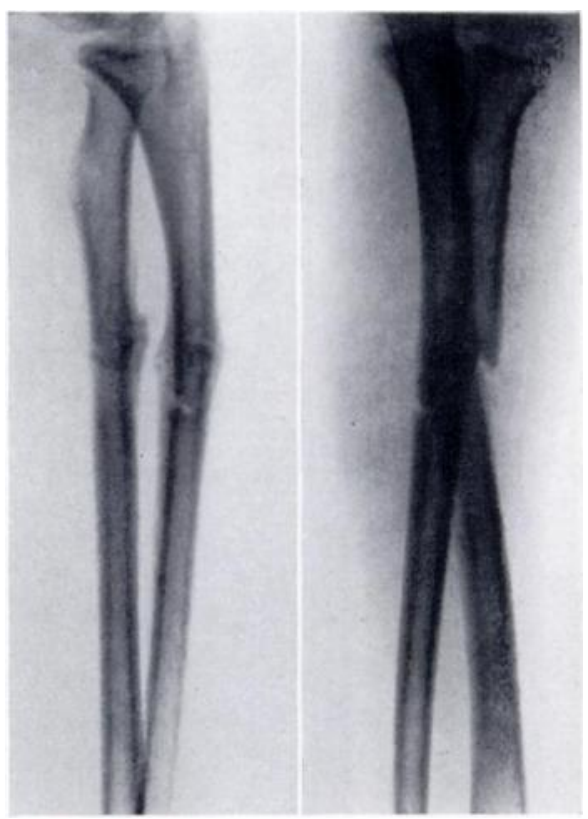

FIG. 7

Union has taken place without loss of position. 
ulnar margin of the bone. As the forearm is pronated the cancellous bone moves laterally until in the midposition it is usually central. 3) The line of the cortical bone on the lateral side-between the head of the radius and the shaft-is quite characteristic. The bone is short and sharply curved in supination and the curve becomes larger and straighter towards mid-rotation. In young children the characteristics of the tuberosity are less clearly defined than in the adult, but the differences in varying degrees of rotation are nevertheless distinct.

Importance of adequate correction of rotational deformity-The need for correcting rotational

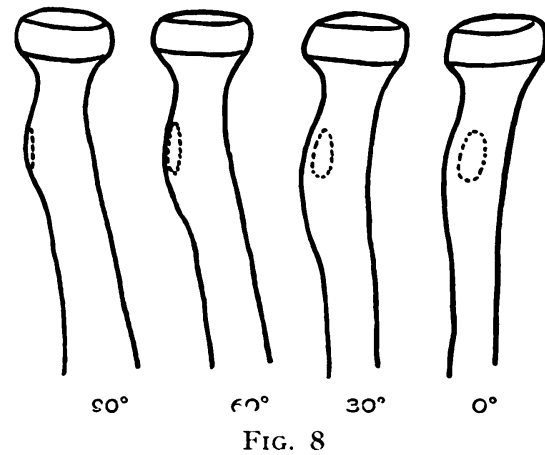

The characteristics of the normal bicipital tuberosity. (See text.) deformity as an essential part of the reduction has already been mentioned. . It has been said that one has only to get the fragments of the radius in perfect alignment to be sure of correcting all rotary displacement but, since this perfect alignment depends upon correct rotation how much more practical to find the rotational position first, when the perfect alignment may be made to follow. The accepted teaching that one should immobilise upper third fractures in full supination and the remainder in the neutral position is unreliable. Figure 9 shows the rotational position of the upper radial fragment in fifty consecutive cases. Although it is true that there is a tendency towards greater supination the higher the fracture, the variations between one case and the next are very considerable; in any case many fractures occur at midshaft level and it is impossible to be certain upon which fragment of the radius the pronator teres is acting.

Correction of the rotational deformity is important also from the point of view of maintaining reduction. The stability of any reduction depends largely on the fit of the bone ends and the more accurate the fit the more stable is the fracture. Jagged bone ends will not fit if they are rotationally displaced. Moreover, rotational deformity means abnormal stresses and strains on soft tissues, and for stable reduction soft-tissue equilibrium is essential. After correction of rotational deformity these fractures can be held in plaster, as will be shown later. Finally, correction of rotation deformity has an important bearing on the functional result. As in many fractures elsewhere, the final range of movement bears a close relationship to the accuracy of the reduction. A fracture that has united with the lower fragment of the

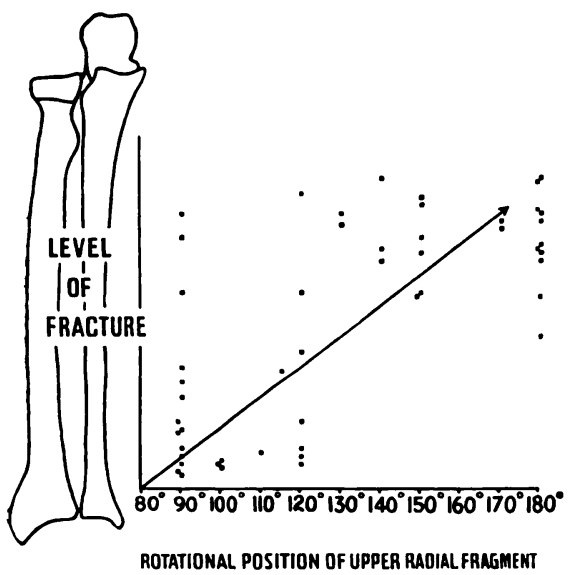

Fig. 9

The rotational position of the upper radial fragment in fifty cases, plotted against the level of fracture Each case is represented by a black dot. radius pronated on the upper will lead inevitably to a corresponding limitation of supination (Figs. 10 and 11). In a review of nineteen cases of fracture of both bones of the forearm treated conservatively over five years ago, it was found that thirteen had been allowed to unite with a pronation deformity of the lower radial fragment of 30 degrees or more, and that in four patients the error was over 60 degrees: in all of the thirteen cases the degree of pronation deformity was reflected in a clinical loss of supination of similar degree. The review showed also that a reduction which may seem excellent as to length and alignment may yet leave a considerable rotation deformity. In a series of forty-one cases treated conservatively Knight and Purvis (1949) found residual rotational deformity of from 25 to 60 degrees in 60 per cent, " the proximal radial fragment being supinated, with

vol. 33 B, No. 4 , November 1951 


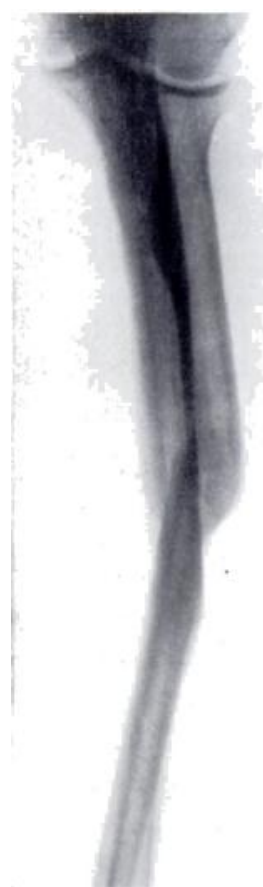

Fig. 10

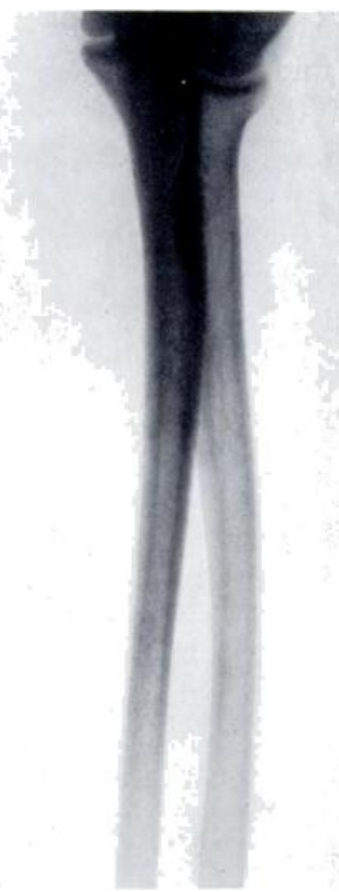

FIG. 11
Figure 10 - An old fracture of both bones of the forearm. The shape of the tuberosity with the hand in neutral rotation corresponds exactly with that of the normal forearm in 60 degrees of supination (Fig. 11), indicating that the fracture has been allowed to unite with a 60 degrees pronation deformity of the lower radial fragment on the upper. Clinically there was loss of supination of 60 degrees. resulting proportional limitation of supination." The disability from persistent rotational deformity-usually pronation of the lower fragment in relation to the upper (Fig. 12) - is such that in recent cases refracture and correction of the rotational deformity may be undertaken to improve the appearance and restore the function of the limb.

Technique of reduction-The fractures are reduced in the usual way by traction and manipulation with the elbow flexed to a right angle. The surgeon should pull on the patient's hand for at least ten minutes against the counter-traction of a sling around the upper arm, and with the forearm rotated into the predetermined position. If on palpation overlap is still found to be present it may be corrected by angulating the forearm while still under traction, in order to engage the bone ends, and then straightening the limb. The position is checked radiographically and the manipulation is repeated if necessary until a satisfactory reduction has been obtained. It is usually possible to secure end-to-end apposition of both bones in this way (76 per cent in the present series). Sometimes the deformity of the radius is difficult to reduce, especially in upper-third fractures. An end-to-end reduction of the ulna, with corner-to-corner reduction of the radius may be accepted, but shortening of the radius should be prevented by thumb traction during the first two weeks and the position must be watched carefully.

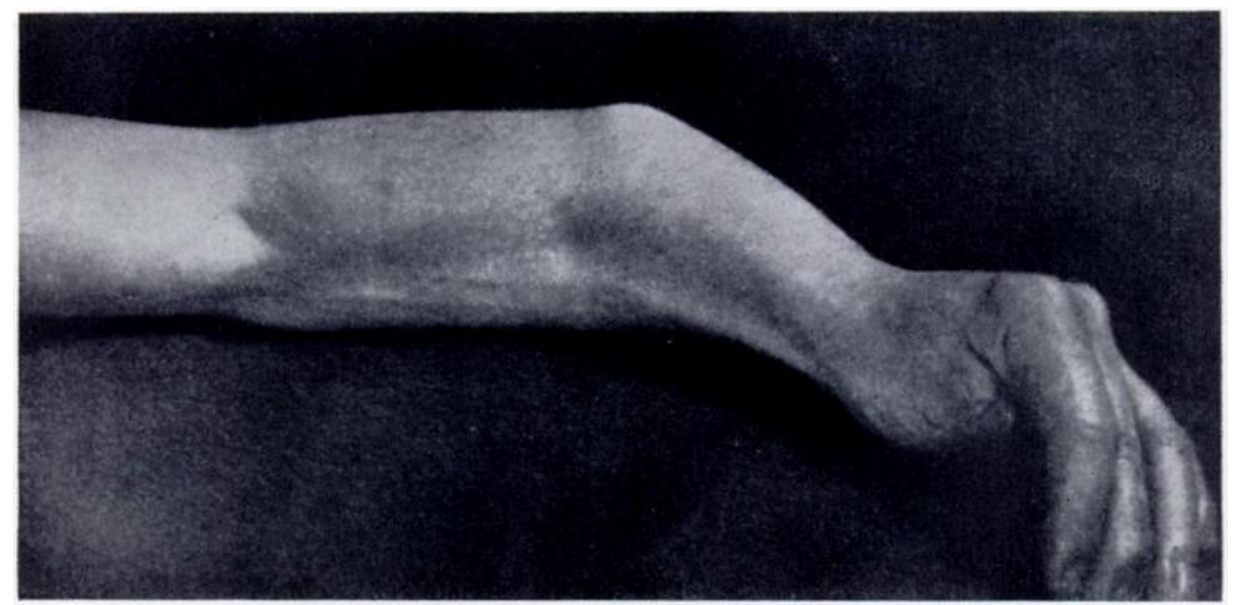

FIG. 12

A severe pronation deformity of the lower radial fragment on the upper. (From Watson-JonesFractures and Joint Injuries). 


\section{FRAGTURES OF THE SHAFT OF THE RADIUS}

As in fractures of both bones of the forearm, complete fractures of the radius are likely to be complicated by rotational displacement of the distal fragment on the proximal, and the deformity must be corrected to ensure accurate reduction and stability (Figs. 13 and 14).

The method of determining the rotational position of the upper radial fragment is precisely the same as that described in the previous section. Certain fractures of the shaft of the radius, however, merit special attention. Where two parallel bones are present, as in the forearm, they form a parallelogram, the one arm of which cannot be shortened without a corresponding shortening of the other. If there is a fracture of the radius with shortening and no fracture of the ulna, there must be radio-ulnar subluxation or dislocation. Cases of this type fall into

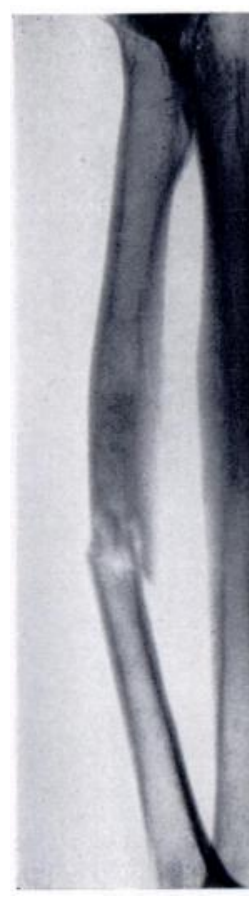

FIG. 13

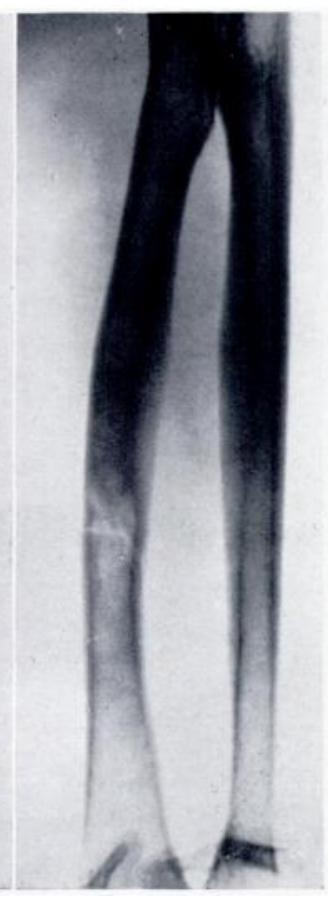

FIG. 14

Fracture of shaft of radius. Figure 13 -Before reduction. Figure 14-Normal alignment restored by correction of rotational deformity.

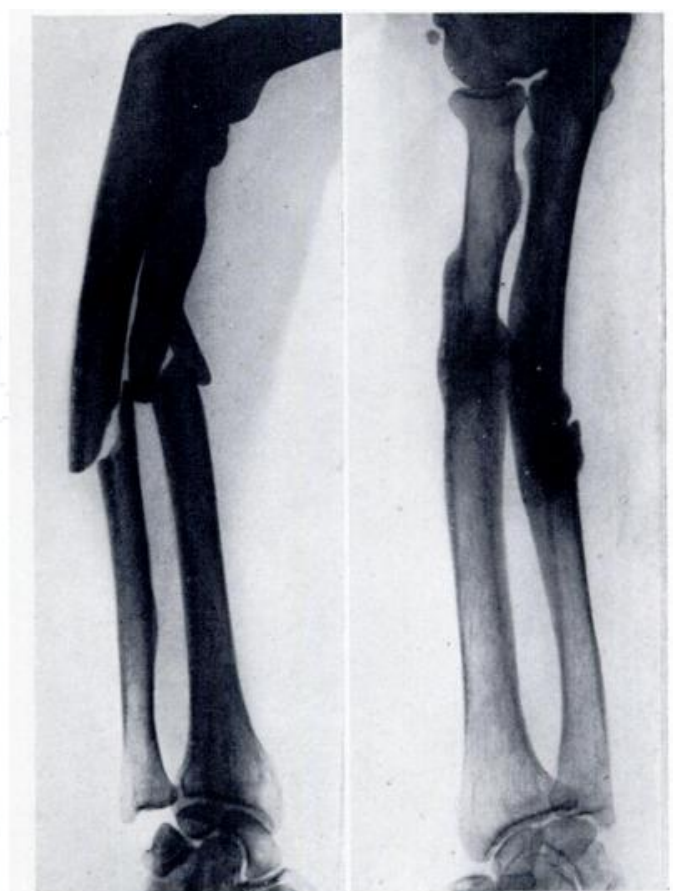

FIG. 15

FIG. 16

Fractures of radius and ulna with comminution of radius. Figure $15-$ Before reduction. Figure $16-$ Shortening of radius prevented by thumb traction.

three groups: 1) Oblique fractures of the shaft, usually in the lower third, with inferior radio-ulnar dislocation or subluxation obvious on the first radiograph. These are unstable fractures and should be treated by primary open reduction and plate fixation. 2) Similar fractures but without obvious subluxation of the inferior radio-ulnar joint on the first examination. 3) Oblique fracture of the radius with an oblique fracture of the ulna running in the same direction as that of the radius. In the last two types there is a tendency for the radius to shorten during the first two weeks and such fractures should be treated by continuous thumb traction through strapping extension attached to a wire loop incorporated in the plaster. Open reduction should be resorted to if shortening of the radius occurs.

Thumb traction-Thumb traction is useful in any injury in which radial shortening is likely to occur, including the types mentioned above. Instability of the radius may be due to: comminution or obliquity of the radial fracture (Figs. 15 and 16); or inability to secure end-to-end reduction of the radial fracture. Thumb traction has been condemned as a cause 
of joint stiffness. The traction need be continued for only two or three weeks, however, and in the present series thumb stiffness was not observed as a complication.

\section{INDICATIONS FOR OPEN OPERATION AND INTERNAL FIXATION}

The indications for internal fixation may be summarised as follows:-

Operation is indicated as a primary procedure:

1) In oblique fractures of the shaft of the radius with inferior radio-ulnar dislocation or subluxation.

2) In compound fractures of both bones with skin loss of such degree that only an immediate skin flap can provide adequate skin cover. In such cases the radius should be plated to give stability to the limb while the more urgent plastic procedure is carried out.

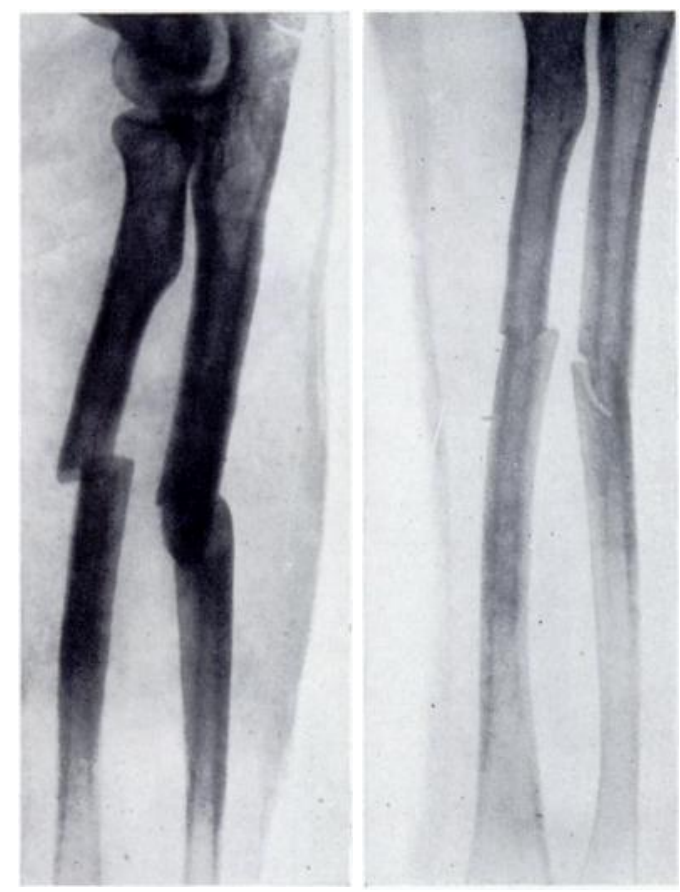

FIG. 17

Fractures of radius and ulna. Note the improvement in alignment obtained by simple correction of rotational deformity.

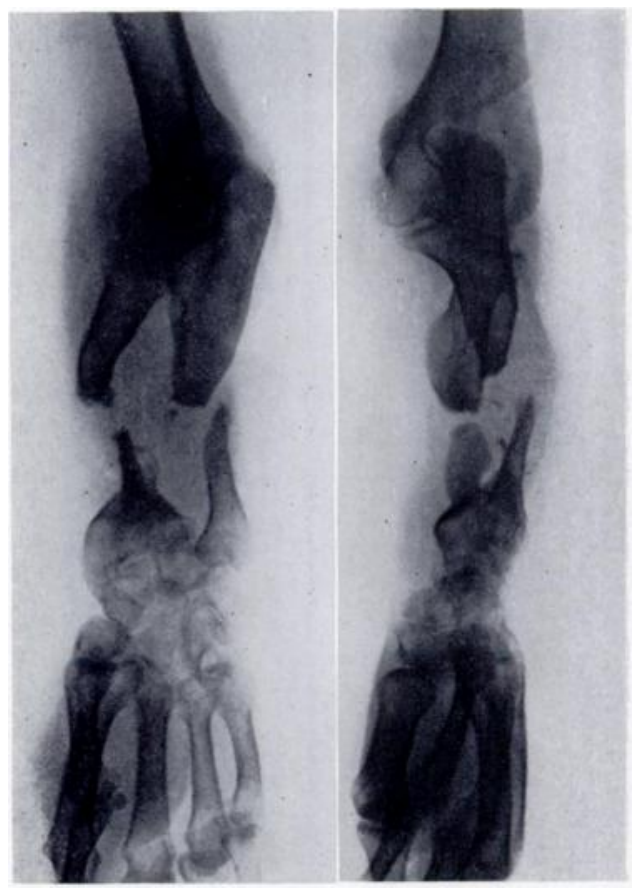

FIG. 18

Originally a simple fracture of radius and ulna. After twenty-two operations the fractures are still ununited.

Operation may be indicated later:

1) In unstable fractures of the radius if radial length cannot be maintained by thumb traction.

2) For delayed union or non-union.

3) If primary reduction has been prevented by severe associated injuries.

It must be admitted that for many surgeons the indications for open operation are much wider than this. It is felt that these fractures are difficult to reduce and hold in plaster and that plate fixation offers a relatively safe way out of the difficulty with, in the recent fracture, the certainty of correcting rotational deformity under direct vision. Such a procedure as a routine, however, is surely a confession of failure. These fractures can and should be treated by closed methods if all elements of the deformity are reduced (Fig. 17). 
Disadvantages of operation-Operative reduction and fixation are difficult, particularly in the upper half of the forearm. Infection, non-union and delayed union do occur (Fig. 18). Holdsworth (1950) reported non-union in 9 per cent and infection in 4 per cent of a series of eighty-five cases. Knight and Purvis (1949) reported non-union in six of thirteen cases treated by open reduction without fixation, and four cases of non-union in twenty treated by plating; they stated also that in several cases rotational alignment had not been restored at the time of operation. Injury to the posterior interosseous nerve is not unknown from a plating operation, and tourniquet paralysis, ischaemic paralysis and contractures are occasional catastrophies. Patrick (1946) considered that the final range of rotation was lessened by the scar tissue of an open reduction and that operation was the commonest cause of crossunion. In short, plate fixation as a routine is not without risk and in any case many of the patients are too young for such treatment to be considered.

\section{RESULTS OF CONSERVATIVE TREATMENT}

How do the results of conservative treatment compare with those of operation? In order to answer this question fifty consecutive fractures of both bones of the forearm have been studied. Only closed complete fractures with overlap have been included, and fractures near the wrist joint have been omitted. The total number of fractures treated during the same period was approximately 7,500 , so that the incidence of closed complete fractures of both bones of the forearm was approximately 1 in 150 . During the same period there were nine compound fractures of both bones and eleven fractures of the shaft of the radius alone.

TABLE I

Rate of Union in Fractures of Both Bones of the Foreari Treated Witholit Operation

\begin{tabular}{|c|c|c|c|c|}
\hline Age & $\begin{array}{c}\text { Number } \\
\text { of } \\
\text { cases }\end{array}$ & $\begin{array}{c}\text { Number } \\
\text { united }\end{array}$ & Complications & $\begin{array}{c}\text { Average } \\
\text { time of } \\
\text { union } \\
(\text { weiks })\end{array}$ \\
\hline $\begin{array}{l}\text { Under } \\
16 \\
\text { years }\end{array}$ & 29 & 29 & - & $7 \cdot 7$ \\
\hline $\begin{array}{l}\text { Over } \\
16 \\
\text { years }\end{array}$ & 21 & 21 & $\begin{array}{c}\text { Malunion } \\
\text { necessitating } \\
\text { bone graft ( } 1 \text { case })\end{array}$ & 14 \\
\hline \multicolumn{5}{|c|}{ Average time of union in 50 cases -10.4 weeks } \\
\hline
\end{tabular}

The treatment in all cases consisted in reduction under general anaesthesia and immobilisation in plaster. The results may be summarised as follows:

Union (Table I)-All the fractures united. In one case the radial fracture collapsed in plaster and the radius was grafted at the seventeenth week; both fractures united within thirty-six weeks. The average time for union in the whole series, including the above case, was $10 \cdot 4$ weeks.

If the patients are divided into those under and those over the age of sixteen years the results are as follows. Under sixteen years-twenty-nine patients; average time for union seven and a half weeks. Over sixteen years-twenty-one patients; average time for union fourteen weeks. (The time of union is considered to be that of external fixation.)

Reduction-End-to-end reduction (that is, more than 50 per cent end-to-end apposition of the bone fragments) of both bones was obtained in thirty-eight patients (76 per cent). Acceptable reduction was obtained in all the remainder.

VOL. 33 B. NO. 4, NOVEMBER 1951 


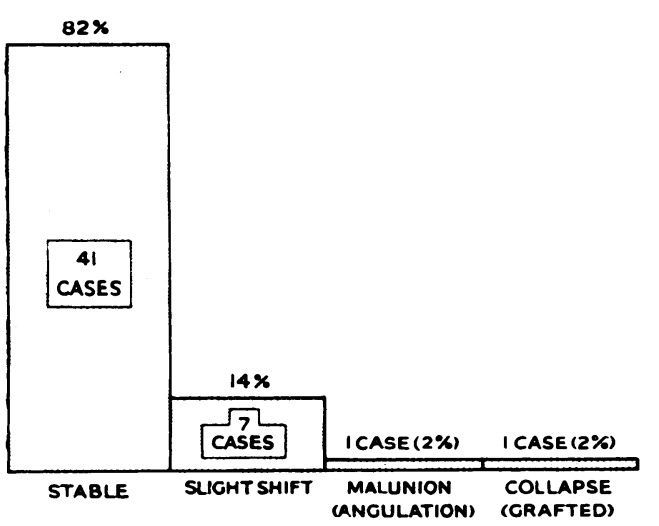

FIG. 19

Behaviour in plaster of tifty fractures of both bones of the forearm treated conservatively.

Behariour in plaster (Fig. 19)-In forty-one patients (8: per cent) the reduced position was held in plaster until the fractures had united. In seven (14 per cent) there was a slight shift in position in plaster not considered sufficient to warrant interference. In one patient (aged seventy-five years) union was slow and both bones angulated in plaster. The functional result was poor (supination 60 degrees, pronation 20 degrees). In one case already mentioned the radial fracture was comminuted and displacement occurred in plaster. Open reduction and bone grafting of the radius were undertaken and union was secured in thirty-six weeks.

Assessment of late rotational deformity-Fortyeight patients were examined radiographically after the fractures had united to determine whether the rotational position estimated at the time of reduction had been correct and had been held in plaster. In forty cases (83 per cent) the rotational position was correct. In seven, there was a residual rotational deformity of less than 30 degrees. In one case rotational deformity was greater than $\mathbf{3 0}$ degrees.

Final range of rotation (Fig. 20)-Of the fifty patients: three had more than 15 degrees limitation of supination; seven had more than 15 degrees limitation of pronation; only four

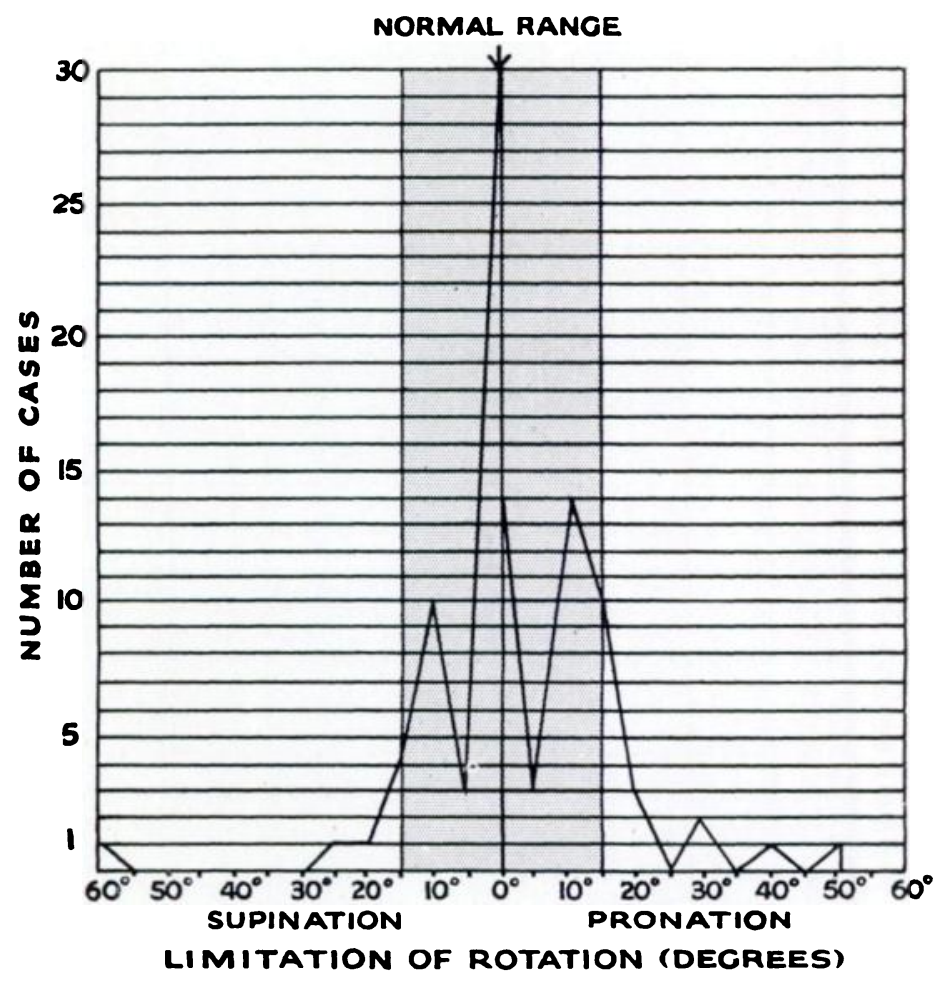

FIG. 20

Graph showing limitation of rotation, as compared with the normal side, in fifty fractures of both bones of the forearm treated conservatively. The shaded section includes all cases with less than 15 degrees limitation of pronation or supination.

THE JOURNAL OF BONE AND JOINT SURGERY 
had more than 30 degrees total limitation of rotational movement. Normal or near-normal function may thus be said to have been regained in over 90 per cent of cases.

Complications-There were no untoward complications in the series.

Discussion--The results show that fractures of both bones of the forearm can be treated by conservative methods with the expectation of union in almost all cases in a reasonably short time and with a return to normal function in most. Quite apart from the complications peculiar to open methods such a claim cannot be made for routine open reduction and plate fixation. For success in conservative treatment, however, it is essential to pay full regard to the rotational element of the deformity.

\section{GREENSTICK FRACTURES OF THE RADIUS AND ULNA}

It has been said that the treatment of greenstick fractures of the radius and ulna presents no problem; nevertheless in a survey of 349 of these fractures treated during 1948 it was found that of the eighty-eight fractures in which the initial deformity was considered worth correcting the deformity recurred to a significant extent in fourteen (16 per cent) (Figs. 21 to 23). It is true that in young children an angular deformity is often only temporary, for a new architecture is soon laid down to restore the bones to normal: it is nevertheless worrying for the parents and disconcerting for the surgeon to find that the deformity has recurred in plaster. These patients were treated along standard lines. The angular deformity was corrected

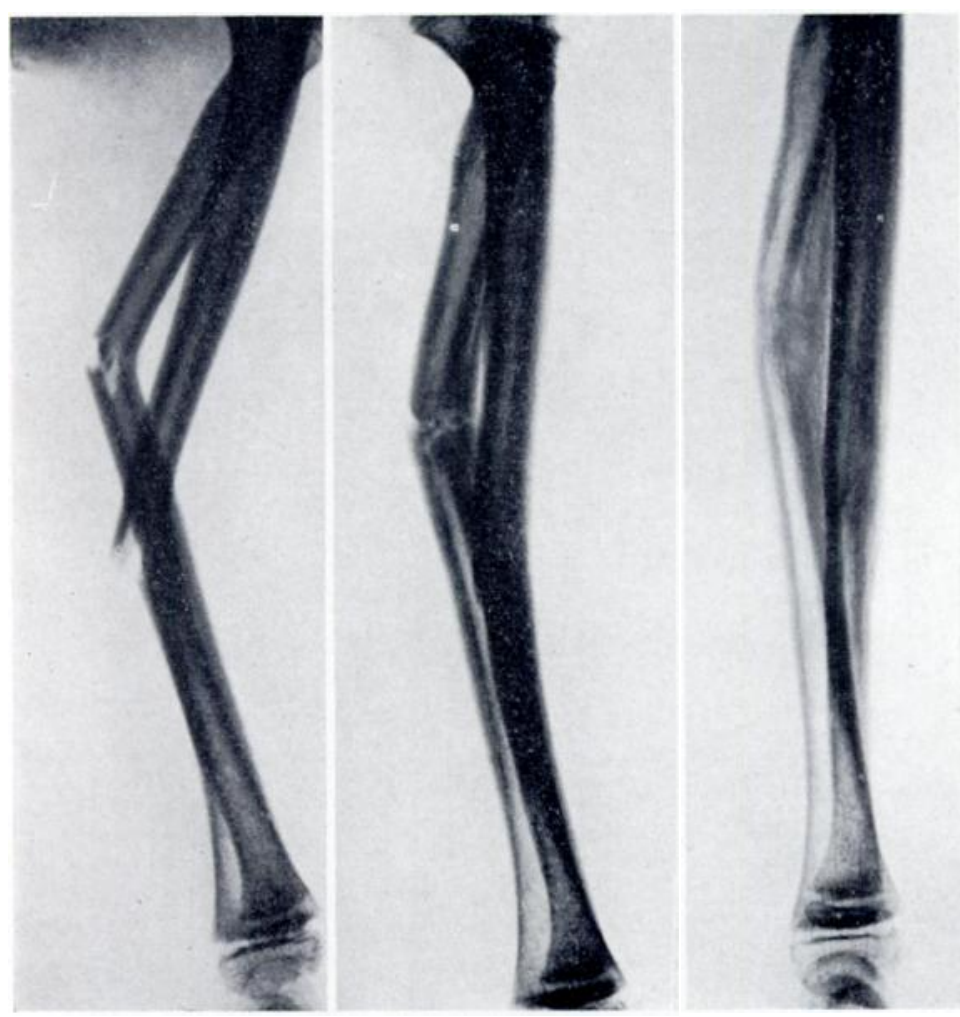

FIG. 21
FIG. 23

Figure 21-A typical greenstick fracture of supination type with forward angulation at the fracture site. Figure 22-The fracture was "reduced" by manipulation in a flat plane. Note that the radial fracture was made complete by the manipulation. Farly union has taken place with recurrence of deformity. Figure 23-Remodelling has largely restored the bone architecture to normal.

vol. $33 \mathrm{~B}$, No. 4, NOVEMBSER 1951 
by manipulation in a flat plane; thus if the limb was bent forwards it was reduced by bending it backwards, and vice versa. It may be noted that this manoeuvre frequently resulted in the conversion of a greenstick into a complete fracture. This has, in fact, been advocated as the only sure way of obtaining complete correction of deformity-surely a violation of one of the basic principles of fracture surgery, that one should not damage intact structures in the reduction. After manipulation the routine procedure was to immobilise the limb in an above-elbow plaster in the position of mid-rotation.

Importance of the rotation element in the causative injury-In all fractures or dislocations the manner in which reduction is obtained is of great importance. Whenever possible the broken structures, bone or soft tissue, should be made to retrace their path, so

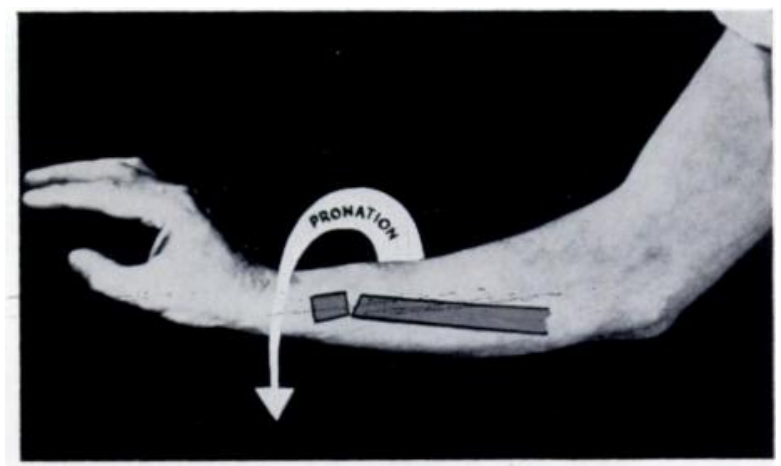

Fig. 24

A fracture sustained while the forearm is pronating will angulate backwards.

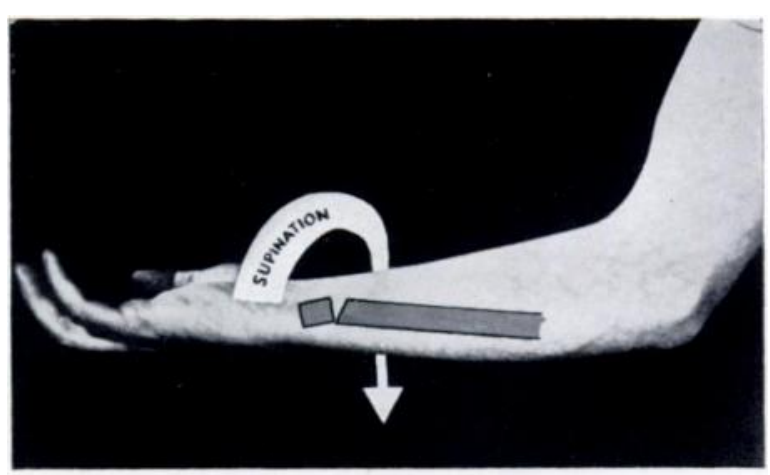

FIG. 25

A supination fracture will tend to angulate forwards.

that no further injury is caused by manipulating the limb " against the grain." This need for care in manipulation calls for a knowledge of the mechanism of the injury, for in general the simplest reduction is a reversal of this mechanism: it is a subject of particular difficulty in the forearm because of the many movements involved, but there are few injuries in which pronation or supination have not played a part. When one falls on to the outstretched hand - the commonest way in which a forearm injury is sustained - the hand becomes fixed on the ground, and above it the momentum of the body will continue to act until equilibrium is reached. The main force acting on the limb will, of course, be vertical compression, but the falling body is hardly likely to be moving solely in a downward direction and some lateral or rotatory element is to be expected in its momentum, to which element 
the forearm will respond by pronation or supination. This movement may go to the extremes of normal anatomy or beyond-in which case a forced rotation injury will result-or, more commonly, will be arrested before these extremes are reached. In either case a rotation violence has been applied to the limb, and the pattern of the fracture and soft-tissue injury will vary accordingly. The differentiation between forced rotation injuries and those sustained while the forearm is pronating or supinating within its normal range is important. In the former group the injuries to be expected can be calculated and there is experimental evidence to support these calculations. In the second group-which may be termed pronation or supination injuries as opposed to forced pronation or forced supination injuries - the picture is not so clear, but it is reasonable to suppose that, if the body is moving in a rotary direction as it falls, this rotation will to some extent determine the direction of displacement and the

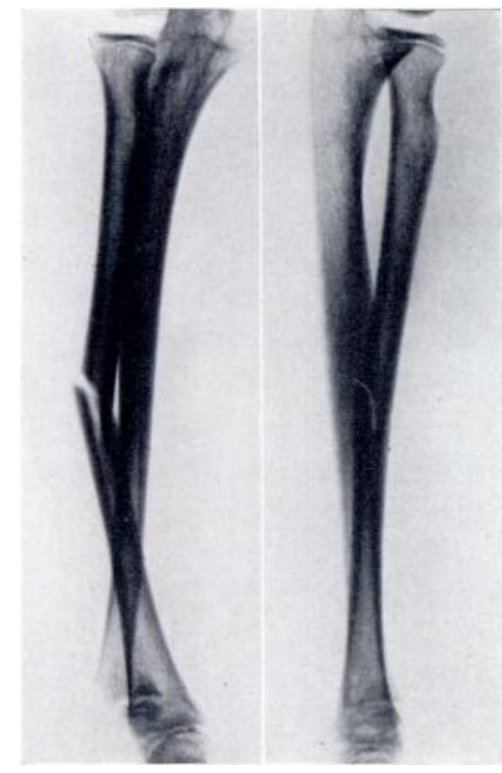

FIG. 26

FIG. 27

Figure 26-Pronation-type greenstick fracture of radius and ulna. Angulation is backwards. Figure 27-Full supination has reduced the deformity.

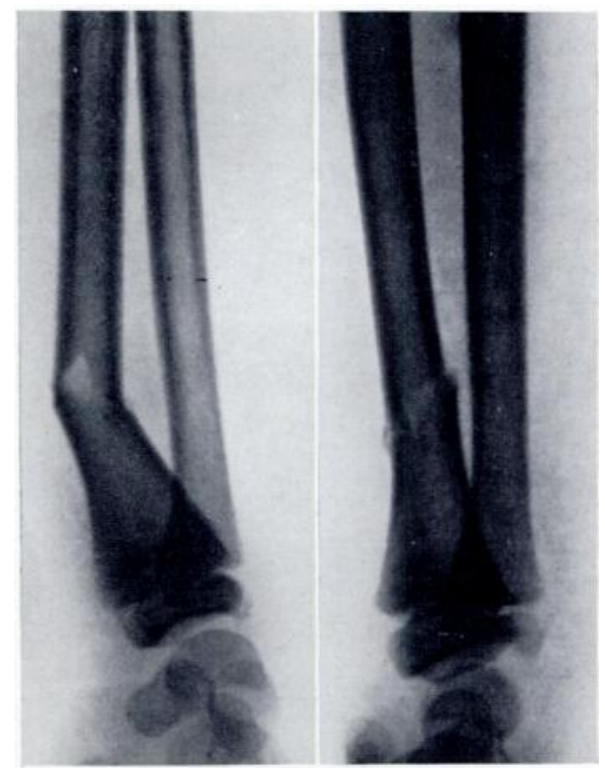

FIG. 28

FIG. 29

Figure 28-Supination-type greenstick fracture of radius. The angulation is forwards. Figure 29 -The fracture has been reduced by full pronation.

nature of the soft-tissue injury. Pronation and flexion are closely allied, both mechanically and developmentally, and a fracture occurring while the forearm is pronating is likely to develop a forward deviation of the distal fragment, with backward angulation at the fracture.* One can demonstrate this by pronating one's own forearm with the other hand; there is in pronation a flexing force which in the presence of a fracture will produce a backward angulation (Fig. 24). Supination and extension are similarly related, and supination fractures will, if they angulate, angulate forwards (Fig. 25). In each case vertical compression supplies the fracturing force but the rotation element decides the direction of angulation. These principles can be most easily illustrated in greenstick fractures of the forearm with

* This Journal follows the usual convention of describing angular deformity of a broken bone as a whole and not of the distal fragment or the proximal. Thus forward angulation (likewise forward bending or bowing) implies that the point (or convexity) of the angle (or bow) is directed forwards; in backward angulation the point of the angle is directed backwards; and so on. When speaking of the direction of the distal fragment, we prefer to avoid ambiguity by speaking of deviation rather than angulation. For instance, if the lower fragment of a broken bone is deviated backwards, the bone as a whole shows forward angulation.

Vol. 33 B, No. 4, November 1951 
angulation, which can be completely corrected by reversing the rotational force that has caused it (Figs. 26 to 29). Furthermore, in these fractures the bones are often broken at different levels and the radius angulated more than the ulna; this would indicate that a rotation force has been acting, for in pronation and supination the radius moves around the static ulna and, if it is fractured while rotating, its deformity would naturally be greater.

This conception makes a new approach to these injuries possible. If, for example, backward angulation is part of the pronation element then the simplest, most effective, and least damaging method of correcting it will be by placing the limb in full supination: the intact soft tissues on the pronation side will then be tightened and will tend to hold the reduction.

In practice the reduction of a greenstick fracture with angular deformity by full pronation or supination, according to whether the angulation is forwards (supination type) or backwards (pronation type), is surprisingly easy (Figs. 26 to 29 ). One has but to grasp the limb at the wrist and fully pronate or supinate as the case may be, and the reduction is complete; there is no undue force, no breaking or tearing of intact soft tissues, no manipulation at the fracture site. The limb is, as it were, a green sapling which has been twisted and has bent to the twist as a sapling will: if the twist is undone the bend disappears and the limb is straight again. If the limb is rotated back again in the opposite direction the angulation can be seen to recur, and to disappear again when the original manoeuvre is repeated. Not only is reduction thus secured more easily and more gently than by the usual method of " correcting " the angulation, but it is more complete, for the intact bone and periosteum of the fragments are restored to their correct rotational alignment. After reduction, the position can be held with comparative certainty by immobilising the limb in an above-elbow plaster in full pronation or supination according to the type of fracture one is dealing with. For example, in a pronation-type greenstick fracture (in which the angulation was backwards) which has been reduced and immobilised in full supination, the deformity cannot recur because the supinators cannot initiate the displacement which the long flexors will develop.

As a practical method of treating all greenstick fractures with angulation the method is open to criticism. Full rotational movement takes longer to return after immobilisation in full pronation or supination; moreover if the method is used as a routine sooner or later a complete fracture of the radial shaft will be so treated with disastrous results, for the fracture will unite with a gross rotational deformity between the two fragments. As a method of reducing these fractures, however, it has much to commend it for it is simple, certain and atraumatic. Immobilisation in the overcorrected rotational position should perhaps be confined to those difficult cases of greenstick fracture with angulation just above the wrist, in which redisplacement is fairly common, and for those cases that have required remanipulation for recurrence of deformity.

\section{CONGLUSIONS}

An attempt has been made to describe some of the ways in which the element of rotation, which is so important a part of the function of the normal forearm, has a bearing upon the mechanism and treatment of forearm injuries. In particular, distinction is drawn between those injuries in which the shaft of the radius remains in continuity, and those in which there is a complete fracture of the bone. In the former, rotation of the hand in the reduction will be transmitted to the upper end of the radius, and extremes of rotational movement may safely be used to obtain and hold a reduction. In the latter there is likely to be a rotational deformity between the two radial fragments, and the lower radial fragment must be placed in accurate rotational alignment with the upper. In the first group reduction, and in certain cases immobilisation, in full pronation or full supination has been shown to have a place in the treatment of those cases in which a rotation violence has shaped the pattern of the injury. Soft tissues may be used to guide and hold a reduction in a rotational injury, just as in injuries of other types. An injury caused by forced pronation should logically be treated in full 
supination, for only thus are the intact soft tissues on the "pronation side" of the limb used to the best advantage.

In the mechanism of injuries caused by rotation violence it is emphasized that vertical compression is usually the basic force, to which a rotation force may be added by the direction of momentum of the body weight. Such injuries may be grouped into forced rotation injuries (in which the violence applied has taken the limb beyond the normal ranges of rotational movement), and injuries occurring while the limb is pronating or supinating. In the latter group the rotational element determines the pattern of the injury: pronation and flexion are closely allied, and a fracture occurring while the forearm is pronating will develop a backward angulation: so also a supination injury will produce a forward angulation.

On the basis of these considerations injuries of the forearm may be classified as follows:

Injuries in which the shaft of the radius remains in continuity

Forced pronation injuries:

1) Forward dislocation of the head of the radius.

2) Backward dislocation of the lower end of the ulna.

3) The anterior Monteggia fracture-dislocation.

These injuries should be reduced and immobilised in full supination to prevent recurrence of deformity. The lateral and posterior Monteggia injuries are probably variants of dislocation of the elbow and are not caused by rotation violence. In general it is considered that all dislocations of the head of the radius are best treated in full supination.

Pronation injuries:

Greenstick fractures of the radius, and of both bones of the forearm, with backward angulation. Reduction of deformity is most easily obtained by manipulating into full supination. Certain fractures may with advantage be immobilised in this position.

Supination injuries:

Greenstick fractures of the forearm with forward angulation. Reduction is best obtained by full pronation.

\section{Injuries in which the shaft of the radius is in two separate fragments}

This group includes all complete fractures of the shaft of the radius and of both bones of the forearm. There is nearly always a rotational deformity between the two radial fragments and its correction is a dominant factor in the treatment.

\section{REFERENCES}

Evans, E. M. (1945): Rotational deformity in the treatment of fractures of both bones of the forearm. Journal of Bone and Joint Surgery, 27, 373.

Evans, E. M. (1949): Pronation injuries of the forearm with special reference to the anterior Monteggia fracture. Journal of Bone and Joint Surgery, 31-B, 578.

Fitzgerald, F. P. (1947): Treatment of displacements of the distal radio-ulnar joint. Proceedings of the Royal Society of Medicine (Section of Orthopaedics), 40, 488.

Holdsworth, F. W. (1950): British Medical Association. Proceedings of the Annual Meeting 1949 (Section of Orthopaedics), 201.

KNight, R. A., and Purvis, G. D. (1949): Fractures of both bones of the forearm in adults. Journal of Bone and Joint Surgery, 31-A, 755.

PAtrick, J. (1946): A study of supination and pronation, with especial reference to the treatment of forearm fractures. Journal of Bone and Joint Surgery, 28, 737.

Watson-Jones, R. (1943): Fractures and Joint Injuries. Third edition. Edinburgh: E. \& S. Livingstone Ltd. 